\title{
ARGUESIAN LATTICES WHICH ARE NOT LINEAR
}

\author{
MARK D. HAIMAN
}

\begin{abstract}
A linear lattice is one representable by commuting equivalence relations. We construct a sequence of finite lattices $A_{n}(n \geq 3)$ with the properties: (i) $A_{n}$ is not linear, (ii) every proper sublattice of $A_{n}$ is linear, and (iii) any set of generators for $A_{n}$ has at least $n$ elements. In particular, $A_{n}$ is then Arguesian for $n \geq 7$. This settles a question raised in 1953 by Jónsson.
\end{abstract}

1. Introduction. A lattice $L$ is linear if it is representable by commuting equivalence relations. Jónsson [6] showed that any such lattice is Arguesian. Numerous equivalent forms of the Arguesian law are now known; it is a strong condition with important applications in coordinatization theory $[\mathbf{1}, \mathbf{2}]$. Nevertheless, the question raised by Jónsson, whether every Arguesian lattice is linear, has remained open until now.

Here we describe an infinite family $\left\{A_{n}\right\}(n \geq 3)$ of nonlinear lattices, Arguesian for $n \geq 7$ (and possibly for $n \geq 4$ ), settling Jónsson's question in the negative. Actually, we obtain more: a specific infinite sequence of identities strictly between Arguesian and linear, and a proof that the universal Horn theory of linear lattices is not finitely based.

2. The lattices $A_{n}$. Let $n \geq 3$. In what follows, all indices are modulo $n$, i.e., $x_{i+1}$ means $x_{0}$ when $i=n-1$, etc. Let $L_{n}$ be the lattice of all subspaces of a vector space $v(\operatorname{dim} v=2 n)$ over a prime field $\mathbf{K}$ with at least 3 elements. Let $\left\{\alpha_{0}, \ldots, \alpha_{n-1}, \beta_{0}, \ldots, \beta_{n-1}\right\}$ be a basis of $v$. Let

$$
\begin{gathered}
m=\left\langle\alpha_{0}, \ldots, \alpha_{n-1}\right\rangle, \quad q_{i}=\left\langle\left\{\alpha_{j} \mid j \neq i\right\}\right\rangle, \quad p_{i}=q_{i} \wedge q_{i+1}, \\
r_{i}=m \vee\left\langle\beta_{i}\right\rangle, \quad s_{i}=r_{i-1} \vee r_{i},
\end{gathered}
$$

where $\langle\cdots\rangle$ denotes linear span. Let

$$
\tilde{A}_{n}=[0, m] \cup[m, v] \cup \bigcup_{i}\left[p_{i}, r_{i}\right] \cup \bigcup_{i}\left[q_{i}, s_{i}\right],
$$

where $[x, y]=\{z \mid x \leq z \leq y\}$.

$\tilde{A}_{n} \subset L_{n}$ is a sublattice; the intervals in the union (2) are its maximal complemented intervals, or blocks; they are the blocks of a tolerance relation on $\tilde{A}_{n}[\mathbf{5}]$; as such, the set $S$ of blocks acquires a lattice structure; specifically, $0_{S}=[0, m], 1_{S}=[m, v], a_{i}=\left[p_{i}, r_{i}\right]$ are atoms, $b_{i}=\left[q_{i}, s_{i}\right]$ are coatoms, and $a_{i}<b_{i}, b_{i+1}$ defines the order relation.

Let $\bar{m}(\operatorname{dim} \bar{m}=n)$ be another vector space, with basis $\left\{\bar{\alpha}_{0}, \ldots, \bar{\alpha}_{n-1}\right\}$. Define $\bar{p}_{i}, \bar{q}_{i}$ by analogy with (1). Let $F=\bigcup_{i}\left[p_{i}, v\right] ; F \subset \tilde{A}_{n}$ is an order filter. Within $F, \bigcup_{i}\left[p_{i}, m\right]$ is an order ideal. Set up a "twisting" isomorphism

Received by the editors March 25, 1986.

1980 Mathematics Subject Classification (1985 Revision). Primary 06C05. 
$\tau$ of $\bigcup_{i}\left[p_{i}, m\right]$ with the order filter $\bigcup_{i}\left[\bar{p}_{i}, \bar{m}\right] \subset[0, \bar{m}]$ as follows: for each $i$, the atoms of $\left[\bar{p}_{i}, \bar{m}\right]$ are of the form $\left\langle r \bar{\alpha}_{i}+s \bar{\alpha}_{i+1}, \bar{\alpha}_{i+2}, \ldots, \bar{\alpha}_{i-1}\right\rangle$ where $(r: s)$ is a ratio of elements of $\mathbf{K}$. Put $\tau\left(\bar{p}_{i}\right)=p_{i}, \tau(\bar{m})=m$, and $\tau\left(\left\langle r \bar{\alpha}_{i}+\right.\right.$ $\left.\left.s \bar{\alpha}_{i+1}, \bar{\alpha}_{i+2}, \ldots, \bar{\alpha}_{i-1}\right\rangle\right)=\left\langle r \alpha_{i}+s \alpha_{i+1}, \alpha_{i+2}, \ldots, \alpha_{i-1}\right\rangle$ except, when $i=0$, put

$$
\tau\left(\left\langle r \bar{\alpha}_{0}+s \bar{\alpha}_{1}, \bar{\alpha}_{2}, \ldots, \bar{\alpha}_{n-1}\right\rangle\right)=\left\langle-r \alpha_{0}+s \alpha_{1}, \alpha_{2}, \ldots, \alpha_{n-1}\right\rangle .
$$

This definition is consistent on $\bar{q}_{i}$ and makes $\tau\left(\bar{q}_{i}\right)=q_{i}$.

Let

$$
A_{n}=F \cup[0, \bar{m}] /(x=\tau(x))_{x \in \bigcup_{i}\left[\bar{p}_{i}, \bar{m}\right]} .
$$

$A_{n}$ is a modular lattice and has the same block decomposition (2) as $\tilde{A}_{n}$, hence the same skeleton lattice $S$. Composing $\tau$ with the automorphism of $[0, \bar{m}]$ induced by the linear transformation $\bar{\alpha}_{1} \mapsto-\bar{\alpha}_{1}, \ldots, \bar{\alpha}_{k} \mapsto-\bar{\alpha}_{k}$, other $\bar{\alpha}_{i}$ fixed, shows that the exceptional interval $\left[\bar{p}_{0}, \bar{m}\right]$ in the definition of $\tau$ could as well have been $\left[\bar{p}_{k}, \bar{m}\right]$, up to an isomorphism of $A_{n}$ respecting the $p_{i}, q_{i}$, $r_{i}, s_{i}$.

\section{Properties of $A_{n}$.}

THEOREM. $A_{n}$ is not a linear lattice.

ProOF. In [3], the author introduced "higher Arguesian identities"

$$
\begin{aligned}
D_{n}: \quad a_{0} & \wedge\left(a_{0}^{\prime} \vee \bigwedge_{i=1}^{n-1}\left[a_{i} \vee a_{i}^{\prime}\right]\right) \\
& \leq a_{1} \vee\left(\left(a_{0}^{\prime} \vee a_{1}^{\prime}\right) \wedge \bigvee_{i=1}^{n-1}\left[\left(a_{i} \vee a_{i+1}\right) \wedge\left(a_{i}^{\prime} \vee a_{i+1}^{\prime}\right)\right]\right)
\end{aligned}
$$

which hold in all linear lattices. $D_{3}$ is the Arguesian law [4]. If we take $a_{i}=p_{i}+\left\langle\beta_{i}\right\rangle$ for all $i, a_{i}^{\prime}=p_{i}+\left\langle\beta_{i}+\alpha_{i}+\alpha_{i+1}\right\rangle$ for $i \neq 0$, and $a_{0}^{\prime}=$ $p_{0}+\left\langle\beta_{0}-\alpha_{0}+\alpha_{1}\right\rangle, D_{n}$ fails in $A_{n}$. In particular, $A_{3}$ is not Arguesian. This minimally non-Arguesian lattice was discovered by Pickering [8].

THEOREM. Every proper sublattice of $A_{n}$ is linear.

ProOF. $\bigcup_{i}\left[p_{i}, r_{i}\right]$ generates $A_{n}$, so a proper sublattice $N \subset A_{n}$ will have $N \cap\left[p_{i}, r_{i}\right] \subset\left[p_{i}, r_{i}\right]$ strictly for some $i$. We can assume $\left[p_{i}, m\right]$ is the exceptional interval in the definition of $\tau$. We show $\left[p_{i}, r_{i}\right]$ (which is a projective plane over K) possesses an automorphism fixing $N \cap\left[q_{i}, r_{i}\right]$ and $N \cap\left[q_{i+1}, r_{i}\right]$ and acting as $\tau$ on $N \cap\left[p_{i}, m\right]$. This is proved by classifying maximal proper sublattices of $\left[p_{i}, r_{i}\right]$ and their possible orientations relative to $m, q_{i}, q_{i+1}$, which leads to 13 cases to check, some trivial, none difficult.

It follows that $\tilde{A}_{n}$ has a sublattice isomorphic to $N$, so $N$ is linear.

THEOREM. If $X \subseteq A_{n}$ generates $A_{n}$, then $|X| \geq n$.

ProOF. For each $j, 0_{S} \cup 1_{S} \cup \bigcup_{i \neq j} a_{i} \cup \bigcup_{i \neq j} b_{i}$ is a sublattice of $A_{n}$ because $\left\{0_{S}, 1_{S}\right\} \cup\left\{a_{i}, b_{i} \mid i \neq j\right\}$ is a sublattice of $S$. For each $j$, therefore, some $x_{j} \in X$ is an element of $a_{j} \cup b_{j}$ and not an element of any other block. This requires $n$ distinct elements of $X$. 
4. Conclusions. The results of $\S 3$ imply that no finite set of identities, or even universal Horn sentences, can completely characterize linearity; in particular, the Arguesian law is insufficient, since it holds in $A_{n}$ for $n \geq 7$. It is known, however, how to characterize linear lattices by an infinite set of universal Horn sentences $[\mathbf{3}, \mathbf{7}]$.

If, as appears likely, the identity $D_{n-1}$ holds in $A_{n}(n \geq 4)$, we would have that $D_{n-1}$ does not imply $D_{n}$, showing that $\left\{D_{n}\right\}$ forms a hierarchy of progressively strictly stronger linear lattice identities. We remark that generator-counting will not suffice for this, since $A_{n}$ has a set of generators $X$ with $|X|=n+3$. We conjecture $n+3$ is minimal, which would imply $A_{4}$ is Arguesian.

\section{REFERENCES}

1. A. Day, Geometrical applications in modular lattices, Universal Algebra and Lattice Theory: Proceedings, Puebla 1982. Lecture Notes in Math., vol. 1004, Springer-Verlag, Berlin and New York, 1983, pp. 111-141.

2. A. Day and D. Pickering, The coordinatization of Arguesian lattices. Trans. Amer. Math. Soc. 278 (1983), 507-522.

3. M. Haiman, Proof theory for linear lattices, Advances in Math. 58 (1985), 209-242.

4. - Two notes on the Arguesian identity, Algebra Universalis 21 (1985), 167-171.

5. Ch. Herrman, S-Verklebte Summen von Verbänden, Math. Z. 130 (1973), 255-274.

6. B. Jónsson, On the representation of lattices, Math. Scand. 1 (1953), 193-206.

7. - Representation of modular lattices and of relation algebras, Trans. Amer. Math. Soc. 92 (1959), 449-464.

8. D. Pickering, On minimal non-Arguesian lattice varieties, Ph.D. Thesis, Univ. of Hawaii, 1984.

Department of Mathematics, Massachusetts institute of TechnolOGY, CAMBRIDGE, MASSACHUSETTS 02139 
